\title{
Compliance with local diabetic guidelines at a district hospital in KwaZulu-Natal, South Africa
}

\author{
K Rampersad* (D, S Rangiah and M Kendon \\ Department of Family Medicine, University of KwaZulu-Natal, Durban, South Africa \\ *Corresponding author, email: kamalrampersad4@gmail.com
}

Background: Diabetes mellitus (DM) represents a major health-related problem in South Africa and throughout the world. The management goals of diabetes are first to maintain normal blood glucose levels and second to prevent the development of complications. Local guidelines developed by the Society for Endocrine Metabolism and Diabetes South Africa (SEMDSA) have shown that tight glycaemic control and appropriate monitoring can prevent or delay the development of diabetic complications. The demographic profile of patients with type $2 \mathrm{DM}$ and the compliance of doctors to the guidelines were determined.

Methods: Five hundred records of patients with type 2 DM were selected from the medical outpatients' department (MOPD) by systematic sampling. Demographic information on age, sex and ethnicity was obtained. The performance and timing of recommended investigations were recorded and compared with the 2012 SEMDSA guidelines.

Results: The mean age of patients was 61 years. Black and Indian patients formed the majority, comprising $44.4 \%$ and $43.0 \%$ respectively. Glycated haemoglobin was measured in $29.2 \%$ of patients once and $13.2 \%$ of patients twice in the past year. Lipid studies were done on $40.4 \%$ of patients. A serum creatinine ( $\mathrm{sCr}$ ), estimated glomerular filtration rate (eGFR) and serum potassium were done on $38.2 \%$ of patients. Eye examinations were done on $13.60 \%$ patients and examination of the foot was done on $7.8 \%$ of patients. Some $15 \%$ had a urine dipstick test done at least once in the past year and $10.4 \%$ had a urine albumin/creatinine ratio (ACR) requested. Only 21 patients (4.2\%) were compliant with the SEMDSA guidelines. Measurements of blood pressure and blood glucose were $100 \%$ compliant. Anthropometric measurements (height, weight and body mass index), dietitian referral and foot examinations were the least compliant, being performed $4.2 \%, 5.0 \%$ and $7.8 \%$ of the time respectively.

Conclusion: Black and Indian patients formed the majority of the study population. The screening for chronic complications of type 2 DM was poor in the majority of patients. Evaluation of selected records demonstrated compliance with the SEMDSA guidelines in only $4.2 \%$ of patients. There is an urgent need to review barriers to the implementation of guidelines in South Africa.

Key words: Diabetes mellitus, adherence, guidelines

\section{Introduction}

Diabetes mellitus (DM), like many other non-communicable diseases, represents a major health-related problem throughout the world. ${ }^{1}$ The 2015 report released by the International Diabetes Federation (IDF) showed that there are currently around 451 million people affected with diabetes worldwide and this is predicted to increase to over 693 million by $2045 .^{2}$

Type $2 \mathrm{DM}$ is the most commonly diagnosed form of diabetes and accounts for more than $90 \%$ of people living with diabetes. ${ }^{3}$ Historically DM was thought to be very uncommon in Africa, however some epidemiologists predict that the economic impact of diabetes, as well as the consequent death toll, will surpass the ravages of HIV and AIDS in the near future. ${ }^{4}$ Currently more than 22 million people within Africa have diabetes. ${ }^{3}$

In South Africa there were 2.3 million cases of diabetes in 2015 with a national prevalence of $7.0 \%{ }^{5} \mathrm{DM}$ is common amongst all race groups with the highest prevalence amongst the Indian population (15.8\%), followed by the Black (4.8\%) and White (3.5\%) populations. ${ }^{3,6}$

Long-term cardiovascular, renal, neurological and retinal complications of type 2 DM are major causes of morbidity and mortality. ${ }^{1-6}$ These complications impact negatively on the patient, his/her family and the community in which he/she resides and works.

Diabetes is the fourth leading cause of death in the world., ${ }^{3,6,7}$ In 2015 approximately 5.0 million people died from diabetes worldwide. The 2015 IDF estimated that there were 57319 diabetic-related deaths in the South Africa. ${ }^{2}$ The mean health expenditure per person was approximately US\$ 918.9.5

The primary goals in the management of type $2 \mathrm{DM}$ are first to maintain normal blood glucose levels throughout the day and second to prevent the development of complications by a systematic and standardised screening programme. ${ }^{5,7}$

DM is a major concern for National Health Systems worldwide because of its impact on morbidity and mortality. Routine measurements such as blood glucose, haemoglobin A1c $(\mathrm{HbA} 1 \mathrm{c})$, lipid determination, blood pressure measurement, annual eye and albuminuria screening have proved to be effective in identifying and treating patients at risk. ${ }^{3,5,7}$

The Society for Endocrine Metabolism and Diabetes of South Africa has developed guidelines based on a number of local and international trials, which showed that tight glycaemic control leads to a reduction in the development of diabetic 
complications. ${ }^{5,7}$ These guidelines are targeted at the resourcelimited South African context and all required tests and examinations are readily available in both the public and private sector. They were first published in 2009 with the aim of providing guidance on the most appropriate management for people with diabetes mellitus and its complications at primary health care level. They are reviewed regularly at 3-4-year intervals and the information is freely available to healthcare professionals by means of journal publications such as the Journal of Endocrinology, Metabolism and Diabetes of South Africa, various online publications and seminar presentations.

Several studies, however, both in developed and developing countries, have shown poor compliance with local guidelines by doctors caring for patients with diabetes. ${ }^{1,6,7}$ A study conducted in Italy in 2003 reviewed 31104 persons with diabetes and concluded that only $20 \%$ of patients received adequate screening for complications as per their recommended local guidelines. ${ }^{1} \quad$ Uganda, a low-income developing country, reported only $14 \%$ of patients had their lipid profiles done annually. In addition only $12.4 \%$ of patients were screened for diabetic nephropathy, no patient was assessed for microalbuminuria and similar poor results of screening for retinopathy, peripheral vascular disease and cardiac complications were identified. ${ }^{8}$ Two studies published in 2013 conducted in the North West province and KwaZulu-Natal reported similar poor compliance with the locally recommended SEMDSA guidelines. ${ }^{9,10}$ Studies conducted at district hospitals providing generalist services, which remain the entry point for the majority of patients to primary health care, are severely lacking.

A large number of patients with diabetes are seen at Northdale Hospital (NH) medical outpatients' department (MOPD) for review and prescribing of their chronic medication. They are seen at six-monthly intervals or more often as requested by the MOPD doctors. There are no statistics available on the demographic profile of these patients. NH has an on-site laboratory, dietetics department and an eye clinic. Many patients, however, are admitted from the MOPD or the emergency department with complications arising from type 2 DM such as strokes, heart attacks and renal failure. Many other patients are referred to the ophthalmologist with advanced stages of diabetic retinopathy. The high occurrence of potentially preventable complications has prompted this study to assess the compliance of healthcare providers with local diabetic guidelines. Observational analysis has shown that recommended screening linked with a reduction in the $\mathrm{HbA} 1 \mathrm{c}$ resulted in several benefits. For every $1 \%$ reduction in the $\mathrm{HbA} 1 \mathrm{c}$, there was a $14 \%$ reduction in myocardial infarction, a $37 \%$ reduction in microvascular complications and a $21 \%$ reduction in deaths related to DM. ${ }^{5}$

The objectives of the study were to review the demographic profile of patients with type $2 \mathrm{DM}$ presenting to an MOPD at a district hospital and to review compliance of doctors with the 2012 SEMDSA guidelines in terms of performing recommended examinations and investigations.

\section{Methods}

The study was performed in a large district hospital in the uMgungundlovu health district, KwaZulu-Natal. The hospital provides medical services to a predominantly low-to-middle income population with a high prevalence of diabetes and hypertension. The MOPD sees approximately 2450 patients with diabetes per year and receives referrals from 12 clinics. It is staffed mainly by medical interns and junior medical officers.
A study sample of 500 patients' charts was reviewed, representing $20 \%$ of the 2450 patients. This is a descriptive study that does not test any statistical hypothesis; the issue of power was not considered. This study included patients who were diagnosed with type $2 \mathrm{DM}$ and were attending the MOPD for more than one year. Patients with type $1 \mathrm{DM}$ or gestational diabetes were excluded from the study due to differing monitoring practices and a lower prevalence in the community.

Systematic random sampling was achieved by randomly assigning a number between 1 and 5 to the charts. Every fifth record was chosen until 500 charts were selected. These records were subsequently assessed against the 2012 SEMDSA guidelines.

The following were assessed: demographic data, presence of hypertension, height, weight, body mass index (BMI), waist circumference, blood pressure, comprehensive foot examination, comprehensive eye examination, blood glucose, $\mathrm{HbA} 1 \mathrm{c}$, lipid profile, sCr, eGFR, potassium, urine dipstick, urine albumin/creatinine ratio $(A C R), E C G$, referral to dietitian and current therapy. Results were recorded as either performed or not performed and the number of times performed if applicable.

The data collected were captured on an Excel $^{\circledR}$ spreadsheet (Microsoft Corp, Redmond, WA, USA) and subsequently analysed using the Statistical Package for the Social Sciences (SPSS; IBM Corp, Armonk, NY, USA). Descriptive statistics such as percentages were used to summarise categorical data.

Permission to conduct the study was obtained from the management of NH, UKZN Biomedical Research Ethics Committee (BE551/16) and the KZN Department of Health. Confidentiality of patients' information was maintained by excluding their names and file numbers during the processing of data.

\section{Results}

There were 296 females (59.20\%) and 204 males (40.80\%). The mean age was 61 years (42-91 years), 222 (44.40\%) of the patients were Black, 215 (43\%) were Indian, 34 (6.8\%) were Coloured and 29 (5.8\%) were White. A total of 275 (55\%) were also receiving treatment for hypertension and $82(16.2 \%)$ patients were receiving insulin therapy (Actraphane ${ }^{\mathrm{TM}}$ or Protophane $\left.{ }^{T M}\right)$, with the balance being managed with oral anti-diabetic agents (Metformin and Glibenclamide or Gliclazide) (Table 1).

Screening tests for complications as per the guidelines were not performed consistently (Table 2). Of the 500 patients, only 21 had a weight, height, BMI and waist circumference recorded. The eye examination was done in only 68 (13.60\%) patients. In

Table 1: Demographic profiles and disease characteristics

\begin{tabular}{llll}
\hline Parameter & \multicolumn{1}{c}{ Categories } & N & $\%$ \\
\hline Sex & Male & 204 & 40.8 \\
& Female & 296 & 59.2 \\
Race & Black & 222 & 44.4 \\
& Indian & 215 & 43 \\
& White & 34 & 6.8 \\
& Coloured & 29 & 5.8 \\
Treatment & Oral therapy only & 418 & 83.8 \\
& Insulin therapy & 82 & 16.2 \\
Presence of hypertension & Hypertension present & 275 & 55 \\
& Hypertension not present & 225 & 45 \\
\hline
\end{tabular}

$N$ : given as a number; \%: percentage. 
Table 2: Screening tests for complications

\begin{tabular}{llr}
\hline Test performed & \multicolumn{2}{c}{$\begin{array}{c}\text { Compliance with the 2012 } \\
\text { SEMDSA Guidelines }\end{array}$} \\
\hline Height & $n$ & $\%$ \\
Weight & 21 & 4.2 \\
BMI & 21 & 4.2 \\
Waist circumference & 21 & 4.2 \\
Blood pressure & 21 & 4.2 \\
Blood glucose & 500 & 100 \\
Eye examination & 500 & 100 \\
\hline Foot examination & 68 & 13.6 \\
HbA1c annually & 39 & 7.8 \\
HbA1c biannually & 146 & 29.2 \\
Lipids & 66 & 13.2 \\
sCr and eGFR & 202 & 40.4 \\
Urine dipstick & 191 & 38.2 \\
Urine ACR & 62 & 12.4 \\
\hline ECG & 52 & 10.4 \\
Dietitian & 64 & 13.2 \\
\hline
\end{tabular}

$n$ : given as a number; \%: percentage.

addition, patients receiving insulin were more likely to be referred for an annual eye examination (50 of the 68 patients) compared with those on oral therapy only.

Examination of the foot was recorded in 39 (7.8\%) patients. Older patients with a mean age of 58.2 years were more likely to have this examination performed.

An HbA1c was done on 146 (29.2\%) patients once in the year and only $66(13.2 \%)$ patients had an HbA1c done twice in the preceding year. Both males and females were equally likely to have this test requested with a male:female ratio of 1:1.12. A lipid analysis including total cholesterol, low density lipoprotein, high density lipoprotein and triglycerides was done on 202 $(40.4 \%)$ patients. A serum creatinine (SCr), eGFR and serum potassium were done on 191 (38.2\%) of patients.

Seventy-six (15.2\%) had a urine dipstick test done at least once in the past year and $52(10.4 \%)$ had a urine ACR requested. An ECG was done on 64 (13.2\%) occasions and only 25 (5\%) patients had been reviewed by the dietitian.

There were no statistical differences in tests performed in relation to age and sex (Tables 3 and 4).

All patients had their blood pressure and blood glucose checked at every visit. The observations are routinely performed by nurses prior to patients consulting with doctors.

Table 3: Relationship between sex and tests performed

\begin{tabular}{lcccc}
\hline Parameter & Males & Females & Chi-squared & $p$-value \\
\hline HbA1c & 92 & 120 & 1.0646 & 0.5873 \\
Lipogram & 89 & 114 & 1.1062 & 0.2929 \\
u-ACR & 27 & 36 & 0.0476 & 0.8272 \\
Eye test & 33 & 36 & $1, .316$ & 0.2513 \\
\hline
\end{tabular}

u-ACR: urine albumin creatinine ratio.

HbA1c: Glycated haemoglobin.
Table 4: Relationship between age and tests performed

\begin{tabular}{lcccc}
\hline Parameter & $\begin{array}{c}\mathbf{4 0 - 5 9} \text { years } \\
\boldsymbol{n}=\mathbf{2 6 4}\end{array}$ & $\begin{array}{c}\mathbf{6 0} \text { years } \\
\text { and more } \\
\boldsymbol{n}=\mathbf{2 3 6}\end{array}$ & Chi-squared & $\boldsymbol{p}$-value \\
\hline HbA1c & 115 & 97 & 7.21 & 0.081 \\
Lipogram & 112 & 91 & 6.8551 & 0.0767 \\
u-ACR & 36 & 37 & 2.1887 & 0.5342 \\
Eye test & 35 & 34 & 2.1997 & 0.5546 \\
\hline
\end{tabular}

$n$ : number of patients.

HbA1c: glycated haemoglobin.

u-ACR: urine albumin creatinine ratio.

\section{Discussion}

In this study, females formed the majority of the patients with a female to male ratio of 1.45:1.

The overall mean age was 61 years. Black and Indian patients represented the majority of the sample population at $44.4 \%$ and $43 \%$ respectively.

This study demonstrated the poor adherence of medical personnel to the SEMDSA guidelines and poor screening of the longterm complications of type $2 \mathrm{DM}$.

Nurse-driven examinations such as recording of blood pressure and blood glucose were performed routinely. However, height, weight, BMI, abdominal circumference and urine dipstick were done with less frequency. This was surprising as these tests require little time, equipment or skill. Other similar studies showed varying results with a South African study reporting a more than $90 \%$ compliance with these bedside tests whilst another African study showed less than $50 \%$ compliance. ${ }^{10,11}$ There was no identifiable reason for their omission in this study. It is possible that a lack of understanding of their significance in the guidelines may have contributed to their omission.

Laboratory tests including $\mathrm{HbA} 1 \mathrm{c}(29.2 \%)$, Scr, eGFR and potassium (38.2\%), and lipids (40.4\%) were performed more frequently but well below recommendations. Studies done in KwaZuluNatal (KZN) and North West province showed better results with $30-90 \%$ of investigations being performed. ${ }^{9,10}$ The presence of an on-site laboratory and phlebotomist simplifies the process for doctors to request blood tests. Doctors may not always be familiar with the guidelines and thus not order recommended tests timeously.

Examinations requiring more clinical skills, including a comprehensive foot examination (7.8\%) and eye examination (13.6\%), were done less frequently. These results mirrored the 2012 study in KZN, which showed only $6 \%$ of patients received a foot exam. ${ }^{10}$ More specialised diabetic centres however, seem to place greater emphasis on examination of the foot with a Ugandan study reporting a $75 \%$ uptake of this test. ${ }^{8}$ Time constraints due to high patient load and uncertainty of clinical skills may have contributed to these outcomes.

Systemic hypertension was common in this study sample with $55 \%$ of patients being managed for both their hypertension and type $2 \mathrm{DM}$ at the MOPD. Although this did not fall under the specific objectives of this study, it is well documented that hypertension is an important modifiable risk factor for both microvascular and macrovascular disease. ${ }^{5,7,12}$ Patients with diabetes and hypertension are therefore more likely to develop target organ damage. $5,7,11,12$ 
A 2009 study published in the Annals of African Medicine showed that the major predictor of resource use in type $2 \mathrm{DM}$ patients in developing countries was the presence of complications as well as inadequate glycaemic control. ${ }^{11}$

Diabetic retinopathy is possibly the most common microvascular complication of diabetes and is responsible for approximately 10000 new cases of blindness every year in the USA.,12-14 Studies in Africa have recorded the prevalence of diabetic retinopathy to range from $7 \%$ in Kenya to $63 \%$ in South Africa. ${ }^{3,7,10}$ A 2010 survey conducted in Cape Town showed that diabetic retinopathy was responsible for $8 \%$ of blindness and $11 \%$ of severe visual impairment. ${ }^{15}$ Loss of vision in patients with diabetes is disabling as it may impair their ability to selfmonitor blood glucose and administer insulin. In addition, it puts them at greater risk of falling and leads to loss of independence. ${ }^{5,7}$ Early identification of retinopathy and appropriate management has been shown to prevent or slow the progression to visual impairment and blindness. ${ }^{7,15}$ Only $13.6 \%$ of patients received an annual eye examination. This finding is unexpected as there is an on-site eye clinic with a senior medical officer in ophthalmology.

Diabetic nephropathy is the leading cause of renal failure in the USA. ${ }^{3,5,7}$ The estimated prevalence in South Africa is $14-16 \% .^{4-6}$ As many as $7 \%$ of patients with type 2 DM may already have microalbuminuria at the time of diagnosis. ${ }^{10}$ In the United Kingdom Prospective Diabetes Study (UKPDS), the incidence of microalbuminuria was $2 \%$ per year in patients with type 2 DM and the 10-year prevalence after diagnosis was $25 \% .{ }^{16}$ Diabetic patients with microalbuminuria typically progress to proteinuria and overt nephropathy if appropriate interventions are not initiated early in the course of the disease. ${ }^{5,7,16,17}$ In a resource-limited area such as South Africa, diabetic nephropathy is a major concern as therapeutic options are limited once a patient reaches the stage of renal failure. A urine dipstick, urine ACR and Scr converted into an eGFR are the recommended investigations by SEMDSA to screen for diabetic nephropathy., ${ }^{4,6}$ Only $15.2 \%$ of patients in this study had a urine dipstick done once in the year, $10.4 \%$ had a urine ACR and $40.4 \%$ had a Scr and eGFR done. A 2009 study conducted in a teaching hospital in Nigeria showed similar poor results with urinalysis and serum creatinine done only $50 \%$ of the time. ${ }^{11}$

Foot examinations and education on foot care is an essential step in diabetic care. It is aimed at identifying persons at risk for ulceration and lower extremity amputation. ${ }^{5,7}$ A thorough examination of the peripheries should be done initially and at least annually thereafter. ${ }^{5,7,17}$ The skin, bones, nerves and vasculature should be assessed. Screening for peripheral neuropathy is done with a monofilament or $128 \mathrm{~Hz}$ tuning fork. It should include testing for sensation to light touch, vibration and temperature. Abnormalities in more than one test of peripheral sensation are $>87 \%$ sensitive in detecting the presence of peripheral neuropathy. $5,7,13$ Patients who have lost 10-g monofilament sensation are at risk of developing foot ulceration., In the NH MOPD, only 39 (7.8\%) patients had documentation of a foot examination being performed. There was, however, no mention of the use of a monofilament or tuning fork and no grading of risk documented. A 2008 study in the Western Cape and a 2011 study in KwaZulu-Natal showed similar poor outcomes with $11.3 \%$ and $6 \%$ of records documenting foot examinations respectively. ${ }^{9,10}$

Routine measurements such as blood glucose, HbA1c, lipid determination, blood pressure measurement, annual eye examination and albuminuria screening have proved to be effective in identifying and treating patients at risk, thereby preventing or delaying the progression of target organ damage. ${ }^{3,5,7,13}$ The early detection of abnormalities is crucial and assists the healthcare provider to decide if treatment needs to be modified, further investigations are warranted or referral to a specialist is indicated. Overall, in this study, compliance with the guidelines was disappointing, which puts patients at a higher risk of developing potentially avoidable complications.

\section{Limitations of the study}

This was a retrospective study with small numbers. The data assessment tool only identified whether investigations were performed; it did not comment on any specific values or appropriateness of actions if results were found to be abnormal.

\section{Conclusions and recommendations}

Compliance with the SEMDSA guidelines for screening of patients with type $2 \mathrm{DM}$ was poor. This could lead to the late identification of complications, resulting in poor outcomes for the patient and an increased demand on health services.

Recommendations from this study are as follows:

- A quality improvement process should urgently be put in place to identify the reason for the discrepancy between current practice and recommended evidence-based practice.

- Continuing medical education should be ensured with emphasis on the SEMDSA guidelines to healthcare workers managing patients with diabetes.

- A simplified data collection tool should be developed and attached to the file of each patient being managed for DM. This should specify the type and frequency of investigation required to ensure that all processes of care are followed.

- With the high prevalence of type 2 DM in the community being serviced by $\mathrm{NH}$, a diabetic clinic in consultation with all stakeholders should be implemented to further improve care for patients with diabetes.

- Patients should be empowered through the principles of Diabetes Self-Management Education and Support.

Acknowledgements - The authors thank Ms Fikile Nkwanyana for statistical support and Dr T Nayager for support throughout the entire study.

Disclosure statement - No conflict of interest was reported by the authors.

Authors' contribution - KR was responsible for the project design, data collection and presentation of results. SR made major contributions and corrections during the project design and writing of the manuscript. MK made corrections during the writing of the manuscript.

\section{ORCID}

K Rampersad (D) http://orcid.org/0000-0003-1592-2975

\section{References}

1. Giorda C, Picariello R, Nada E, Tartaglino B, Marafetti L, Costa G, et al. The impact of adherence to screening guidelines and of diabetes clinics referral on morbidity and mortality in diabetes. PLoS One. 
2012;7(4):e33839 [Online]. [cited 2015 Oct 2]. Available from: https:// doi.org/10.1371/journal.pone.0033839

2. Cho NH, Shaw JE, Karuranga S, Huang $Y$, da Rocha Fernandes JD, Ohlrogge AW, et al. IDF diabetes atlas: global estimates of diabetes prevalence for 2017 and projections for 2045. Diabetes Res Clin Pract. 2018 Apr;138:271-81. [cited 2018 Jul 20]. https://doi.org/10. 1016/j.diabres.2018.02.023

3. International Diabetes Federation. Epidemiology and morbidity, diabetes and impaired glucose tolerance; global burden; prevalence and projections, 2014 and 2040. 2015. [cited 2015 Nov 3]. Available from: https://www.oedg.at/pdf/1606_IDF_Atlas_2015_UK.pdf.

4. Piotie PN. Diabetes epidemic in Africa: now is the time to act, Part 1. Consultancy Africa Intelligence Public Health Unit 2013. [cited 2015 Nov 15]. Available from: http://www.inonafrica.com.

5. The society for endocrinology, metabolism and diabetes of South Africa type 2 diabetes guidelines expert committee. The 2017 SEMDSA Guideline for the Management of Type 2 Diabetes Guideline Committee. JEMDSA 2017;21(1) (Supplement 1):S1-S196.

6. Kalk WJ, Pick WM, Sayed AR. Diabetes mortality in South Africa. S Afr Med J. 1998;88:1259-62.

7. Society for endocrinology, metabolism and diabetes of South Africa: guidelines for the diagnosis and management of type 2 diabetes mellitus for primary health care 2012. [cited 2015 Nov 3]. Available from: http://www.semdsa.org.za.

8. Kibirige D, Atuhe D, Sebunya R, Mwebaze R. Suboptimal glycaemic and blood pressure control and screening for diabetic complications in adult ambulatory diabetic patients in Uganda: a retrospective study from a developing country. J Diabetes Metab Disord. 2014;13 (1):40-7. https://doi.org/10.1186/2251-6581-13-40

9. Tumbo JM, Kadima FN. Screening of long-term complications and glycaemic control of patients with diabetes attending Rustenburg
Provincial Hospital in North West Province, South Africa. Afr J Prim Health Care. 2013;5(1):375-80.

10. Igbojiaku OJ, Harbor OC, Ross A. Compliance with diabetes guidelines at a regional hospital in KwaZulu-Natal, South Africa. Afr J Prim Health Care Fam Med. 2013;5(1):447-52. https://doi.org/10. 4102/phcfm.v5i1.447

11. Agaba El, Puepet FH, Ugoya O, Agaba, PA, Adabe R, Duguru M, et al. Chronic kidney disease screening and renoprotection in type 2 diabetes. Ann Afr Med. 2009;8(1):52-4. https://doi.org/10.4103/15963519.55765

12. Fowler MJ. Microvascular and macrovascular complications of diabetes. Clin Diabetes. 2008;26(2):77-82. https://doi.org/10.2337/ diaclin.26.2.77

13. Conradie M, Myburgh K, Lombard CJ, Hugh S. Complications of diabetes in the white and coloured populations of the cape. S Afr Med J. 1998;88(12):1262-4.

14. Puoane T, Steyn K, Bradshaw D, Laubscher R, Fourie J, Lambert V, et al. Obesity in South Africa: the South African demographic and health survey. Obes Res. 2002;10:1038-48. https://doi.org/10.1038/ oby. 2002.141

15. Hofman KJ, Cook C, Levitt N. Preventing diabetic blindness: a priority for South Africa. S Afr Med J. 2014;104(10):661-2. https://doi.org/10. 7196/SAMJ.8580

16. King P, Peacock I, Donnelly R. The UK Prospective Diabetes Study (UKPDS): clinical and therapeutic implications for type 2 diabetes. $\mathrm{Br} J$ Clin Pharmacol. 1999;48(5):643-8. https://doi.org/10.1046/j. 1365-2125.1999.00092.x

17. Rheeder P. Type 2 diabetes: the emerging epidemic. $S$ Afr Fam Pract. 2006;48(10):20-21. 